\title{
Longitudinalidade/continuidade do cuidado: identificando dimensões e variáveis para a avaliação da Aten ção Primária no contexto do sistema público de saúde brasileiro
}

\author{
Longitudinality/continuity of care: identifying dimensions \\ and variables to the evaluation of Primary H ealth Care \\ in the context of the Brazilian public health system
}

Elenice M achado da Cunha ${ }^{1}$

LigiaGiovanella ${ }^{2}$

${ }^{1}$ Fundação O swaldo Cruz. Av. Brasil 4.365/318,

M anguinhos. 21040-360

Rio deJaneiro RJ.

elenice@ensp.fiocruz.br

${ }^{2}$ Departamento de

Administração e

Planejamento em Saúde,

Escola Nacional de Saúde

Pública Sergio Arouca

Fundação O swaldo Cruz.
Abstract Longitudinality, which is concerned with Primary Health Care (APS) professionals accompanying patients over time, is considered a central feature of this level of health care. The fulfilment of thisattributeis related to positivehealth results, which justify its use for assessing Primary $\mathrm{H}$ ealth Care. On the other hand, the term is not commonly used by Brazilian authors, and in international literaturetheterm "continuity of care" is used with a similar meaning. Therefore, this study is composed of a conceptual revision of longitudinality/continuity of care, as well as theidentification of its dimensions so as to enable the actual performance of this attribute to be assessed. Asa result, the similarity between the two terms is highlighted, although the identified dimensions of each attribute do not entirely coincide. The revision allowed three dimensions to be adopted in accordance with the context of the Brazilian public health care system: identification of the basic unit as regular source of care, a long-lasting treatment bond and continuous information. The proposal of an APS evaluation variables presented herein is line with these dimensions.

Key words Primary health care, Longitudinality/continuity of care, Assessment in primary care
Resumo A longitudinalidade, quetrata do acompanhamento do paciente ao longo do tempo por profissionais da equipe de atenção primária em saúde (APS), é considerada característica central destenível assistencial. 0 atendimento a este atributo está relacionado com resultados positivos, 0 que justifica sua utilização para fins de avaliação da APS. Por outro lado, o termo não éusual entre os autores brasileiros, e na literatura internacional o termo continuidade do cuidado é utilizado com sentido semelhante. 0 presente estudo consiste em revisão conceitual sobre a longitudinalidade/continuidade do cuidado, bem como a identificação de suas dimensões, de forma a favorecer a avaliação do atendimento ao referido atributo. Como resultado, destaca-se a semel hança entre os termos, embora as dimensões identificadas para 0 atributo não sejam totalmente coincidentes. $\mathrm{A}$ revisão permitiu a adoção de três dimensões em acordo com o contexto do sistema de saúde público brasileiro: identificação da unidade básica como fonte regular de cuidado, vínculo terapêutico duradouro econtinuidadeinformacional. A proposta de variáveis para a avaliação da APS aqui apre sentada vai ao encontro dessas dimensões.

Palavras-chave Atenção primária em saúde, Longitudinalidade/ continuidadedo cuidado, Avaliação na atenção primária 


\section{Introdução}

A atenção primária em saúde (APS) tem sido consideradaimprescindível para a efetividadedos sistemas de saúde e para a garantia de melhorias nas condições de saúde da população. Vários estudos comprovam que países que possuem sistemas organizados a partir da APS apresentam menores taxas de incidência de doenças e de internação, redução de taxas de mortalidade prematura por causas evitáveis, menores custos e maior equidade na oferta de serviços ${ }^{1-3}$.

Embora haja consenso com relação à importância da APS, o mesmo não ocorre no que diz respeito à sua definição. Além do mais, formas de organização e operacionalização destenível assistencial são bastante diferenciadas nos diversos países ${ }^{4}$. N esse sentido, Starfield ${ }^{5}$ tem influenciado outros autores ao afirmar que a atenção primáriase diferencia dos outros níveis assistenciais por apresentar quatro atributos: atenção ao primeiro contato, longitudinalidade, integralidadeecoordenação. Tais atributos tem sido utilizados para estruturar instrumentos que buscam avaliar a qualidade da APS nos Estados Unidos e no Brasil $6^{6-10}$.

Dos atributos identificados por Starfield ${ }^{5}, a$ longitudinalidade tem sido considerada característica central e exclusiva da APS. Para esta auto$\mathrm{ra}$, trata-se do acompanhamento do paciente ao longo do tempo por médico generalista ou equipe de APS, para os múltiplos episódios de doença ecuidados preventivos. N esteacompanhamento, está implícita uma relação terapêutica caracterizada por responsabilidade por parte do profissional de saúde e confiança por parte do paciente $^{11,12} .0$ atendimento a tal atributo tende a produzir diagnósticos e tratamentos mais precisos, além da redução dos encaminhamentos desnecessários para especialistas e para a realização de procedimentos de maior complexidade ${ }^{11,13}$.

Em artigos relativosà APS, muitas vezeso termo "continuidade do cuidado" é utilizado com significado semelhante ao de longitudinalidade. $M$ as para Starfield ${ }^{5}$, esses dois termos possuem significados diferentes, uma vez que a continuidade do cuidado estaria relacionada a um problema de saúde específico ea sucessão de eventos entre uma consulta e outra, bem como aos mecanismos de transferência de informação para subsidiar decisões com relação ao tratamento do paciente, sem a preocupação com o estabelecimento de uma relação terapêutica ao longo do tempo.

Assim como Starfield ${ }^{5}$, Pastor-Sánchez et al. ${ }^{14}$ também diferenciam longitudinalidade de continuidade do cuidado. Entendem a longitudinali- dade como o acompanhamento dos distintos problemas de saúde por um mesmo médico, e a continuidade do cuidado como o acompanhamento por um mesmo médico ou não, de um problema específico do paciente. Ressaltam ainda que a continuidade não éum elemento característico da atenção primária, nem exige uma relação pessoal entre o profissional e o paciente, uma vez que bons registros podem suprir a ne cessidade de informação para o devido acompanhamento da patologia.

No Brasil, as reformas que culminaram com a implantação do SUS buscaram fortalecer a APS e ampliar a sua cobertura. Todavia, embora dados comprovem o aumento progressivo da oferta de ações e serviços nesse nível de atenção, os resultados encontrados nem sempre são satisfatórios ${ }^{15,16}$.

Cumprida a etapa de aumento considerável da cobertura da APS, concentram-se os esforços para melhoria da sua qualidade. N este sentido, algumas iniciativas de caráter governamental constituem-seem incentivos para a realização de avaliações internas e externas ${ }^{17}$. M as, instrumentos que avaliem os processos utilizados para alcançar efetividade neste nível assistencial ainda são raros e pouco sensíveis.

N este aspecto, métodos que avaliem 0 atendimento da "Iongitudinalidade" podem ser oportunos, posto que tal atributo está relacionado à efetividade naAPS. Por outro lado, a palavra longitudinalidadenão éusual na literatura nacional, tampouco há consenso quanto a sua definição e forma demedição. Sendo assim, esteestudo consiste em revisão conceitual sobre a longitudinalidade na APS. Tem por objetivo a definição do termo, bem como a identificação das dimensões do atributo, de forma a favorecer a eleição de variáveis que sejam passíveis de medição. A expectativa é acrescentar elementos na discussão sobre monitoramento e avaliação da atenção primária no contexto brasileiro.

\section{M étodo}

Para melhor definir o termo longitudinalidade e identificar suas dimensões, a opção foi por desenvolver revisão bibliográfica sobre a temática. A revisão teveinício a partir deartigos etextosindicados por especialistas da área. Em seguida, deu-se a busca por outros artigos, tanto a partir das referências citadas pel os autores inicial mente consultados, quanto nos bancos de dados do M EDLINE, SciELO e LILACS. Para esta busca, realizada durante os meses de dezembro de 2006 e janeiro 
de 2007, utilizou-se como descritores os termos "Iongitudinalidade" e"continuidade do cuidado", vinculados ao termo "atenção primária".

Para a seleção dos artigos, foram considerados os seguintes critérios: estudos ou análises críticas relativas à longitudinalidade e ou continuidade do cuidado na APS e a disponibilidade do artigo completo de forma gratuita. Foram excluídos trabal hos relacionados ao tratamento de patologias específicas e acompanhamento do paciente por outros profissionais não médicos.

Enfim, para a presente revisão, foram considerados dezessete artigos: quatro indicados edisponibilizados por especialistas previamente consultados; doze a partir de busca em banco de dados e um fornecido pelo autor (Quadro 1). Além destes textos, três obras dos autores citados como referência conceitual nos artigos revisados foram consultadas (Starfield ${ }^{5}, \mathrm{M}$ cW inney ${ }^{18}$ e Institute of M edicine ${ }^{19}$ ).

Os textos revisados foram organizados em três grupos. 0 primeiro contemplou os autores cujo foco éa definição dos termos e identificação das suas dimensões. Os pressupostos conceituais de Starfield servem de base para a discussão, dado que a obra desta autora encontra-se mais difundida entre os especialistas brasileiros que discutem a temática.

No segundo grupo, estão os autores que desenvolvem análise crítica com relação à temática no contexto das reformas setoriais, sem que necessariamenteexplicitem uma referência conceitual. No terceiro, estão os autores que utilizam metodologia específica a fim de mensurar a longitudinalidade e ou continuidade do cuidado e, consequentemente, explicitar seus benefícios (Quadro 2). A proposta de variáveis para a avaliação da atenção primária no contexto brasileiro é contemplada na discussão dos resultados.

\section{Qual a definição delongitudinalidade?}

Do conjunto da literatura investigada, podemos considerar queseis autores buscam definir a longitudinalidade e/ou continuidade do cuidado. Destes, Starfield ${ }^{5}, M$ cW inney ${ }^{18}$ el nstitute of Medicine $\mathrm{e}^{19}$ são publicações conceituais relativas à APS. Os outros são duas revisões sistemáticas (Saultz ${ }^{12}$ e H aggerty et al. ${ }^{20}$ ) e uma análise crítica (Freeman e Hjortdah ${ }^{21}$ ) (Q uadro 1).

Desse primeiro grupo de autores, a revisão sistemática elaborada por H aggerty et al..$^{20}$ constituiseem esforço deconstrução deconsenso com relação ao termo "continuidade do cuidado". Nesta revisão, os autores definem continuidade na APS como relação entre um médico e um paciente que se estende além de episódios espeć́ficos de doença. Esta relação é considerada reflexo de um senso de afiliação, frequentemente expresso em termos de contrato implícito de lealdade por parte do paciente eresponsabilidade dínica por parte do médico. Ressaltam ainda que a afiliação a uma fonteregular decuidado é por vezes referida como continuidade interpessoal e que esta favorece a melhora da comunicação, confiança e senso de responsabilidade.

Outra contribuição do estudo de Haggerty et al. ${ }^{20}$ éa identificação de três tipos de continuidade: informacional, gerencial e relacional. Continuidade informacional éo el emento quepermite a conexão de informação entre diferentes prove dores para a condução do cuidado. Tanto a informação relativa à condição clínica, quanto 0 conhecimento sobre as preferências, valores e contexto do paciente seriam importantes para assegurar 0 atendimento às necessidades do indivíduo. Continuidadegerencial éespecialmente importantena doença clinicamente complexa ou crônica que necessita do envolvimento de vários provedores para a condução do cuidado. Quando existente, os serviços são ofertados de forma complementar e em tempo oportuno. Já a continuidade relacional diz respeito a uma progressiva relação terapêutica entre pacienteeum ou mais provedores ao longo do tempo, que oferece a percepção de garantia do cuidado futuro.

A definição de $\mathrm{Haggerty}$ et al. ${ }^{20}$, por referir quea relação se estendepor mais de um episódio de doença e afirmar o reconhecimento de uma fonteregular decuidado, vai ao encontro do conceito de longitudinalidade para Starfield ${ }^{5}$. Corrobora a hipótese de que estes dois termos são utilizados por muitos autores com sentido semelhante. Por outro lado, essa revisão sistemática acrescenta elementos à discussão ao afirmar que 0 acesso aos serviços de saúde é um prérequisito para a continuidade do cuidado equea coordenação do cuidado éum agente facilitador.

A preocupação com a definição de continuidade do cuidado no âmbito da APS, e em especial pela continuidade interpessoal como uma de suas dimensões, levou Saultz ${ }^{12}$ a investigar o tema utilizando como método a revisão sistemática. Esse autor, que cita como referência conceitual os postulados de $\mathrm{M} \mathrm{CW}$ inney ${ }^{18}$, encontrou como resultado a falta de consenso na definição do termo, mas identificou múltiplas dimensões do mesmo, a saber: informativa, cronológica ou longitudinal, interpessoal, geográfica, interdisciplinar e continuidade familiar. 
Embora tenha destacado essas seis dimensões, Saultz ${ }^{12}$ conclui quecontinuidade do cuidado engloba uma hierarquia de apenas três: informacional, longitudinal e interpessoal. A base hierárquica para a definição do termo seria a con- tinuidade informacional, descrita como "coleção organizada de informação médica e social sobre cada paciente, disponi bilizada para todos os profissionais de saúde que cuidam do mesmo". M as, como o conhecimento sobre o pacientenão seria

Quadro 1. Artigos revisados segundo termo utilizado, interpretação do termo e referência conceitual.

\begin{tabular}{|c|c|c|c|}
\hline Autor & Termo & Interpretação & Referência conceitual \\
\hline $\begin{array}{l}\text { Haggerty et al. } \\
\text { (2003) }\end{array}$ & Continuity of care & $\begin{array}{l}\text { Relação médico-paciente que se estende para além de } \\
\text { episódios específicos. } \\
\text { Dimensões: informacional, gerencial e relacional. }\end{array}$ & \\
\hline Saultz (2003) & $\begin{array}{l}\text { Continuity of care } \\
\text { (Interpersonal } \\
\text { continuity) }\end{array}$ & $\begin{array}{l}\text { Relação longitudinal entre pacientes e aqueles que } \\
\text { cuidam deles. Transcende múltiplos episódios de } \\
\text { doença e inclui responsabilidade por prevenção e } \\
\text { coordenação do cuidado. Envolve confiança e } \\
\text { responsabilidade. } \\
\text { Dimensões: informacional, Iongitudinal, interpessoal. }\end{array}$ & McWinney (1997) \\
\hline $\begin{array}{l}\text { Freeman e } \\
\text { Hjortdahl (1997) }\end{array}$ & Continuity of care & $\begin{array}{l}\text { Cuidado com o mesmo provedor ao longo do tempo } \\
\text { para mais do que um episódio de doença. } \\
\text { Duas interfaces: continuidade longitudinal (médico) } \\
\text { e continuidade pessoal (médico e equipe). }\end{array}$ & Freeman (1985) \\
\hline $\begin{array}{l}\text { Guthrie e Wyke } \\
\text { (2000) }\end{array}$ & $\begin{array}{l}\text { Continuity of care, } \\
\text { personal continuity }\end{array}$ & $\begin{array}{l}\text { Relação médico-paciente assegurando cuidados que } \\
\text { levam em conta o contexto pessoal e social do } \\
\text { paciente (personal continuity). }\end{array}$ & $\begin{array}{l}\text { General Practitioners } \\
\text { Committee (2000) } \\
\text { Freeman e H jortdahl } \\
\text { (1997) }\end{array}$ \\
\hline WHO (1992) & Continuity of care & $\begin{array}{l}\text { Relação contínua entre um usuário e um provedor } \\
\text { individual, uma equipe ou ainda uma rede de serviços. }\end{array}$ & \\
\hline $\begin{array}{l}\text { Ortún e Gervas } \\
\text { (1995) }\end{array}$ & Longitudinalidad & $\begin{array}{l}\text { Prestação de cuidado ao longo do tempo, por médico } \\
\text { de APS, para distintos problemas de saúde. }\end{array}$ & \\
\hline Gérvas (2005) & Longitudinalidad & $\begin{array}{l}\text { Prestação de cuidado ao longo do tempo, por médico } \\
\text { de APS, para distintos problemas de saúde. }\end{array}$ & \\
\hline Christakis (2003) & Continuity of care & $\begin{array}{l}\text { Relação médico-paciente. M édico regular necessário } \\
\text { para o estabelecimento de confiança. }\end{array}$ & $\begin{array}{l}\text { Institute of M edicine - } \\
\text { (1994) }\end{array}$ \\
\hline $\begin{array}{l}\text { Roberge et al. } \\
(2001)\end{array}$ & Longitudinality & $\begin{array}{l}\text { Relação consistente entre paciente e médico ao longo } \\
\text { do tempo - reconhecimento da fonte regular de } \\
\text { cuidado para necessidades de saúde. }\end{array}$ & $\begin{array}{l}\text { Freeman e H jortdahl } \\
\text { (1997) }\end{array}$ \\
\hline $\begin{array}{l}\text { Maeseneer et al. } \\
(2003)\end{array}$ & $\begin{array}{l}\text { Continuity in family } \\
\text { medicine - } \\
\text { longitudinality }\end{array}$ & $\begin{array}{l}\text { Relação pessoal entre médico e paciente ao longo do } \\
\text { tempo. }\end{array}$ & Starfield (1998) \\
\hline $\begin{array}{l}\text { Nutting et al. } \\
(2003)\end{array}$ & Continuity of care & Relação médico-paciente ao longo do tempo. & $\begin{array}{l}\text { Institute of Medicine } \\
\text { (1994) - M cWinney } \\
\text { (1981) }\end{array}$ \\
\hline Fan et al. (2005) & Continuity of care & $\begin{array}{l}\text { Relação entre médico e paciente, caracterizado por } \\
\text { confiança e senso de responsabilidade. }\end{array}$ & Saultz (2003) \\
\hline $\begin{array}{l}\text { Sánchez et al. } \\
\text { (1997) }\end{array}$ & Longitudinalidad & $\begin{array}{l}\text { Acompanhamento dos distintos problemas de saúde } \\
\text { de um paciente por um mesmo médico. }\end{array}$ & Starfield (1992 e 1994) \\
\hline $\begin{array}{l}\text { Gill e M ainous } \\
\text { (1998) }\end{array}$ & Continuity of care & $\begin{array}{l}\text { Acompanhamento pelo mesmo provedor ao longo do } \\
\text { tempo. }\end{array}$ & Starfield (1992) \\
\hline $\begin{array}{l}\text { Saultz e } \\
\text { Albedaiwi (2004) }\end{array}$ & $\begin{array}{l}\text { Interpersonal } \\
\text { continuity of care }\end{array}$ & $\begin{array}{l}\text { Relação duradoura entre médico e paciente } \\
\text { caracterizada por confiança e responsabilidade. }\end{array}$ & Saultz (2003) \\
\hline $\begin{array}{l}\text { Cabana e Jee } \\
(2004)\end{array}$ & $\begin{array}{l}\text { Sustained continuity } \\
\text { of care }\end{array}$ & $\begin{array}{l}\text { Cuidado ao longo do tempo por um único médico ou } \\
\text { equipe de saúde e uma comunicação adequada e } \\
\text { efetiva da informação em saúde. }\end{array}$ & Starfield (1980) \\
\hline $\begin{array}{l}\text { Mainous et al. } \\
\text { (2001) }\end{array}$ & Continuity of care & Relação ao longo do tempo entre médico e paciente. & Starfield (1992) \\
\hline
\end{tabular}


suficiente para assegurar a continuidade do cuidado com um provedor, no segundo nível da hierarquia estaria a continuidade longitudinal que diz respeito ao estabelecimento de uma unidade de saúde onde 0 paciente deve receber a maioria dos cuidados de saúde, de forma que 0 mesmo sinta-se familiarizado com o ambientee o identifique como referência ao longo do tempo. A equipe de saúde, por sua vez, assumiria a responsabilidade de coordenação do cuidado, incluindo serviços preventivos.

Em escala progressiva, viria à continuidade interpessoal, descrita como uma relação médico-paciente, na qual o paciente conhece e confia no médico eo tem como referência básica para a atenção à saúde. 0 médico, por sua vez, se responsabiliza pela continuidade dos cuidados ao longo do tempo. Para Saultz ${ }^{12}$, o el emento essencial da atenção primária é a continuidade interpessoal, cuja ocorrência depende da presença da continuidade informacional e da continuidade longitudinal.

Duas das três dimensões da continuidade citadas por $\mathrm{H}$ aggerty et al. ${ }^{20}$, a saber, continuidade informacional econtinuidaderelacional, seaproximam das dimensões identificadas por Saultz ${ }^{12}$, denominadas respectivamente continuidade informacional e continuidade interpessoal. Quanto à "continuidade gerencial" citada por Haggerty et al. ${ }^{20}$, cujo significado encontra-se mais pró-

Quadro 2. Artigos revisados segundo autores, tipo de estudo e principais resultados.

\begin{tabular}{|c|c|c|}
\hline Autor & Tipo de estudo & Resultados \\
\hline $\begin{array}{l}\text { Guthrie e Wyke } \\
\text { (2000) }\end{array}$ & Análise crítica & $\begin{array}{l}\text { A suposta contradição entre a premência da modernização da clínica e a } \\
\text { continuidade pessoal seria falsa. }\end{array}$ \\
\hline WHO (1992) & Grupo de trabalho & $\begin{array}{l}\text { Relevância da equipe, necessidade de articulação dos provedores, redução do } \\
\text { sobreuso. }\end{array}$ \\
\hline Gérvas (2005) & Análise crítica & Longitudinalidade permite corrigir falsos negativos. \\
\hline $\begin{array}{l}\text { Ortún e Gérvas } \\
\text { (1995) }\end{array}$ & Análise crítica & Longitudinalidade confere eficiência à APS. \\
\hline Christakis (2003) & Análise crítica & $\begin{array}{l}\text { Interpretação dos autores quanto à natureza da continuidade do cuidado, se é } \\
\text { processo ou resultado, interfere nos achados dos estudos. }\end{array}$ \\
\hline $\begin{array}{l}\text { Roberge et } \\
\text { al.(2001) }\end{array}$ & $\begin{array}{l}\text { Técnica de grupos } \\
\text { focais (médicos e } \\
\text { pacientes) }\end{array}$ & $\begin{array}{l}\text { Congruência de percepções. Disponibilidade de tempo e divisão de } \\
\text { responsabilidades faz com que médicos defendam a relação terapêutica com a } \\
\text { equipe de APS. }\end{array}$ \\
\hline $\begin{array}{l}\text { M aeseneer et al. } \\
\text { (2003) }\end{array}$ & $\begin{array}{l}\text { Estudo de coorte ( } 2 \\
\text { anos) }\end{array}$ & $\begin{array}{l}\text { A utilização de uma fonte regular de cuidados de APS leva a menores gastos } \\
\text { em saúde. }\end{array}$ \\
\hline $\begin{array}{l}\text { Nutting et al. } \\
\text { (2003) }\end{array}$ & Estudo transversal & $\begin{array}{l}\text { Pacientes vulneráveis valorizam mais a continuidade do cuidado. Tal fato pode } \\
\text { interferir nos achados dos estudos. }\end{array}$ \\
\hline $\begin{array}{l}\text { Pasto-Sánchez et } \\
\text { al. (1997) }\end{array}$ & $\begin{array}{l}\text { Estudo descritivo } \\
\text { (questionário com } \\
\text { médicos) }\end{array}$ & $\begin{array}{l}\text { O grau de longitudinalidade e de continuidade averiguado a partir da presença } \\
\text { de listas de paciente e do grau de conhecimento sobre o histórico do paciente } \\
\text { nos países investigados são diferenciados. }\end{array}$ \\
\hline $\begin{array}{l}\text { Mainous et al. } \\
\text { (2001) }\end{array}$ & $\begin{array}{l}\text { Estudo tranversal } \\
\text { (inquérito com } \\
\text { pacientes) }\end{array}$ & $\begin{array}{l}\text { A continuidade com o mesmo médico, ao longo do tempo, está relacionada } \\
\text { com o aumento da confiança no médico. }\end{array}$ \\
\hline Fan et al.(2005) & $\begin{array}{l}\text { Estudo transversal } \\
\text { (inquérito com } \\
\text { pacientes e base de } \\
\text { dados) }\end{array}$ & Continuidade do cuidado está relacionada com maior satisfação do paciente. \\
\hline $\begin{array}{l}\text { Gill e M ainous } \\
\text { (1998) }\end{array}$ & $\begin{array}{l}\text { Estudo longitudinal } \\
\text { com beneficiários do } \\
\text { Medicaid ( } 2 \text { anos) }\end{array}$ & $\begin{array}{l}\text { A continuidade do cuidado está relacionada com menores taxas de internação } \\
\text { por condições crônicas sensíveis ao tratamento ambulatorial. }\end{array}$ \\
\hline $\begin{array}{l}\text { Saultz e } \\
\text { Albedaiwi (2004) }\end{array}$ & Revisão sistemática & $\begin{array}{l}\text { Falta consenso na definição e nas formas de medição da continuidade } \\
\text { interpessoal. M as pode-se afirmar que a Continuidade interpessoal está } \\
\text { relacionada com maior satisfação do paciente. }\end{array}$ \\
\hline $\begin{array}{l}\text { Cabana e Jee } \\
\text { (2004) }\end{array}$ & Revisão sistemática & $\begin{array}{l}\text { A longitudinalidade está relacionada com redução da taxa de internação e de } \\
\text { atendimentos de emergência, maior adesão aos cuidados preventivos e melhor } \\
\text { comunicação. }\end{array}$ \\
\hline
\end{tabular}


ximo do atributo da "coordenação" referido por Starfield ${ }^{5}$, não é contemplada por Saultz. Já o termo continuidade longitudinal utilizado por Saultz, cujo significado diz respeito à identificação de uma unidade de saúde como fonte regular de cuidados, é considerado por Haggerty et al. como pré-requisito para a continuidade do cuidado, mas não como dimensão do atributo.

Vale ressaltar que, embora Starfield não explicite dimensões para o atributo em questão, esta autora cita a identificação da fonte regular de cuidados e o vínculo longitudinal como fatores inerentes à longitudinalidade. Já a continuidade informacional é tratada por esta autora no âmbito do atributo da coordenação do cuidado. MacWinney utiliza o termo "continuidade do cuidado" e identifica cinco dimensões para o mesmo: informacional, cronológica, geográfica interdisciplinar e interpessoal; e o Institute of M edicine, que também utiliza o termo "continuidade do cuidado", não cita dimensões.

Freeman e H jortdah ${ }^{18}$ são citados por outros autores como referência conceitual, mas discutem a temática no âmbito das reformas organizacionais e gerenciais da APS. Esses autores confrontam os termos continuidade longitudinal e continuidade pessoal como duas interfaces da continuidade do cuidado. Uma relação terapêutica mais duradoura com um único médico generalista caracterizaria a continuidade longitudinal. Já na continuidade pessoal haveria a re lação duradoura com um generalista, mas também poderia haver uma equipe de atenção primária que dividisse responsabilidades.

Ao confrontar essas duas interfaces da continuidade do cuidado, os autores explicitam vantagens e desvantagens da continuidade longitudinal. Dentre as desvantagens, destacam o fato dessetipo de continuidade exigir mais disponibilidade e compromisso por parte do profissional médico. Além do mais, alegam que a existência deuma relação duradoura não significa necessariamente que ela seja boa.

Outra afirmativa é que a relação duradoura com um único médico poderia interferir no senso de observação dos pacientes e torná-los menos críticos com relação à avaliação dos cuidados recebidos. Por outro lado, esta relação favoreceria o retorno, para o profissional, da eficácia do diagnóstico e do tratamento. Frente a essas questões, a al egação éque a continuidade longitudinal não seria imprescindível, mas a continuidade pessoal, sim. M as, em todo caso, para o estabelecimento da continuidade pessoal, seria necessário um certo grau de continuidade longitudinal.
A partir dos artigos já discutidos, verifica-se que o termo continuidade do cuidado é utilizado com sentido semel hante ao de longitudinalidade definido por Starfield. A concordância diz respeito, principalmente, ao estabelecimento de relação terapêutica duradoura entre paciente e profissionais de saúde da equipe APS. Por outro lado, observa-sediversidade denomenclatura ediscordância na identificação das dimensões do atributo.

\section{A longitudinalidade no contexto das reformas do setor saúde}

Como dito anteriormente, a longitudinalidade tem sido identificada enquanto questão polêmica no âmbito das reformas que implicam reorganização da oferta de serviços. N este aspecto, Guthrie e Wyke ${ }^{22}$, assim como Freeman e Hjortdahl21, também discutiram a temática no processo de reforma da atenção primária, mas no contexto do Reino Unido.

A princípio, Guthrie e Wyke ${ }^{22}$, que compreendem a continuidade como 0 estabelecimento de contato com o mesmo médico ao longo do tempo, distinguem continuidade pessoal - descrita como uma progressiva relação médico-paciente que assegura cuidados que levam em conta o contexto pessoal e social do paciente -, de continuidade do cuidado, que estaria relacionada com a prática moderna da atenção primária, em que haveria a renúncia de uma atenção mais personalizada e duradoura em prol do atendimento por equipes maiores, que assegurassem a continuidadea partir da utilização deguidelinese registros eletrônicos.

$M$ as a suposta polêmica entre esses dois tipos de continuidade em nome da eficiência seria falsa, dada as evidências de que a continuidade pessoal traria vantagens, como a diminuição das internações e da utilização de serviços de emergência, e maior satisfação do paciente. Logo, não haveria necessidade de escolher entre a suposta "medicina moderna" e a continuidade pessoal; ambas seriam compatíveis. No entanto, os mé dicos precisariam ter mais clareza deste fato de modo a reivindicar a continuidade pessoal no âmbito das reformas do setor saúde. Estes autores não utilizam os termos Iongitudinalidade ou continuidadelongitudinal.

O texto da Organização Mundial de Saúde sobre continuidade do cuidado, publicado em $1992^{23}$, também tem como fonte motivadora as mudanças nos sistemas de atenção primária e o risco de tais reformas interferirem negativamen- 
te nos resultados da atenção. Este texto não refere o termo longitudinalidade e a interpretação para continuidade do cuidado é mais operacional do que conceitual, pois define o termo simplesmente como a relação contínua entre o usuário e o provedor de atenção primária. Os benefícios apontados como relacionados à continuidade do cuidado são maior possibilidade de integração das dimensões físicas, psicológicas, sociais eeconômicas; mel hora na relação entreusuários e provedores de serviços; promoção de um papel mais efetivo na manutenção de saúde por parte do usuário; redução do sobreuso dos serviços de saúde eainda provável redução dos custos do cuidado, por prevenir a duplicação dos serviços e tratamentos desnecessários.

A continuidade do cuidado também aumentaria a satisfação do usuário com o serviço e do profissional de saúdecom o seu trabalho. A única desvantagem apontada no texto diz respeito à dificuldadeem identificar serviços dequalidadeduvidosa, particularmente nos sistemas que não permitem a livre escolha de provedor por parte do usuário ${ }^{23}$. Neste aspecto, vale lembrar que 0 modelo de atenção primária quevem sendo imple mentado no Brasil, com adscrição da população à uma equipe de Saúde da Família, de certa forma, restringe o direito de escolha do usuário com relação à eleição da fonte regular de cuidados.

As análises críticas de Ortún e Gérvas ${ }^{13}$ e de Gérvas ${ }^{11}$, já contempladas no início do texto, discutem as vantagens da utilização de um médico generalista como fonte regular de cuidados e de sua função de "filtro" para 0 acesso à atenção especializada, o que estaria relacionado à eficiência na APS. Já Christakis ${ }^{24}$ discute quea interpretação da continuidade do cuidado nos estudos, ora como processo, ora como resultado da atenção, podeinfluenciar métodose achados. D efende que este atributo deve ser considerado como um resultado. Logo, os estudos deveriam estar direcionados para a melhor forma de alcançar o referido atributo na APS. Por outro lado, reconhece que há questões ainda não respondidas, como a legitimidadedeimpor a continuidadecom uma fonte usual de cuidado para aqueles que não a valorizam. Esse autor, que tem como referência teórica o Institute of $M$ edicine ${ }^{19}$, reconhe cea importância da relação médico-pacientepara que haja continuidade do cuidado.

0 estudo de Roberge et al..$^{25}$ não se constitui em referência conceitual de longitudinalidade e nem almeja identificar suas dimensões; logo, não se encontra entre o primeiro grupo de autores, mas se destaca por buscar a compreensão dos fatores queinfluenciam a utilização deum determinado estabelecimento de saúde como fonte regular de cuidados ao longo do tempo. Para alcançar tal objetivo, os autores utilizaram a técnica de grupos focais com médicos e pacientes, explicitando e comparando suas percepções com relação à temática.

Ao tratarem da fidelidade com a fonte de cuidados, Roberge et al. ${ }^{25}$ utilizaram o termo longitudinalidade e o definiram como "relação consistenteentre pacienteemédico quesetraduz em reconhecimento e utilização de uma fonte regular de cuidado ao longo do tempo". O recurso metodológico permitiu identificar quais fatores estão relacionados com essa fidelidade e, consequentemente, com a longitudinalidade.

Os achados desses autores demonstram certa congruência das percepções de médicos e de pacientes com relação aos papéis a serem desempenhados pelos mesmos na manutenção da fidelidade do paciente com a fonte regular de cuidado. Ambos apontaram os seguintes fatores como determinantes: confiança do paciente no médico, disponibilidade e leal dade do médico, e boa comunicação - na qual o paciente busca relatar corretamente seus sintomas eseu tratamento e estar aberto às recomendações. Outra visão congruenteé que o médico deve ser eficaz, coordenar o cuidado e assegurar o tratamento.

Os grupos de pacientes também apontaram a necessidade do médico estar atualizado com relação às novas descobertas e respeitar a opinião do paciente. Com relação ao estabelecimento de vínculo longitudinal, questões como necessidade de maior disponibilidade e excessiva responsabilidade apareceram como justificativa para a defesa, por parte dos médicos, da relação terapêutica com a equipe, enquanto quea preferência dos pacientes é a relação pessoal com o médico.

\section{Como mensurar a longitudinalidade eseus benefícios}

0 problema da falta de uniformidade na definição de continuidade do cuidado implica a dificuldadeem generalizar resultados. Além do mais, a forma de percepção da temática interfere no tipo de pesquisa, nas formas de coleta de dadose ainda nos resultados do estudo ${ }^{24}$. Nos artigos revisados que buscaram mensurar a continuidade do cuidado (Quadro 2), também foi verificada diversidade na interpretação do termo e, consequentemente, das unidades de análise. No en- 
tanto, a maioria dos estudos indicou associação entre continuidade do cuidado e resultados positivos, como diminuição da taxa de internações e maior satisfação do paciente, constatados nos estudos analisados a seguir.

Para investigar a relação entre custos e continuidade do cuidado, De M aeseneer et al..$^{26}$ utilizaram como recurso metodológico um estudo de coorte por um período de dois anos. Compararam dois grupos de ben eficiários de duas companhias de seguro. Um grupo tinha um generalista como fonte regular de cuidado na atenção primária e o outro, não.

Tendo Starfield como referência conceitual, esses autores interpretaram o termo como relação pessoal entre médico e paciente ao longo do tempo. Como resultado, após ajustes por fatores sociodemográficos, econômicos e presença de comorbidade, esses autores encontraram forte associação entre continuidade do cuidado com médico de família e menores gastos em saúde. Ou seja, os resultados do estudo indicaram que a longitudinalidade estava associada com a eficiêncianaAPS.

Partindo do pressuposto que a valorização da continuidade do cuidado podeinfluenciar na satisfação do paciente com relação à atenção recebida, Nutting et al. ${ }^{27}$ investigaram tal associação em um estudo transversal. Os autores definiram a continuidade do cuidado como um aspecto fundamental da atenção primária ea relacionaram com a identificação de um médico generalista como fonte regular de cuidados. As referências conceituais foram o Institute of $M$ edicine ${ }^{19} \mathrm{eM}$ cW hinney ${ }^{18}$.

No esforço para medir o alcance desse atributo, os autores privilegiaram tópicos relativos ao conhecimento médico sobre o paciente, a coordenação do cuidado e a comunicação interpessoal, além do grau de valorização da continuidade do cuidado pelos pacientes, pressupondo quetal valorização exerce influência na avaliação desse atributo por parte do paciente. Os dados foram coletados a partir de observação direta einquérito com pacientes. Dados sociodemográficos e do estado de saúde percebido pelo paciente serviram de base para a análise.

Os resultados do estudo de Nutting et al. ${ }^{27}$ indicaram que mulheres, idosos, grupos com menor nível de escolaridade, portadores de condições crônicas, segurados do M edicare e M edicaid e a percepção de pior estado de saúde estavam associados com a valorização da continuidade do cuidado. A conclusão desse estudo é sugestiva de que a continuidade do cuidado deve ser assegurada principalmente para grupos de pacientes crônicos, portadores de patologias complexas, idosos e população de baixa renda e quetalvez pessoas jovens esaudáveis não tenham tanto interesse em manter um vínculo com uma fonte regular de cuidados.

O bjetivando medir a longitudinalidade e a continuidade em quatro países europeus, a saber, Espanha, Portugal, Finlândia e Suécia, Pastor-Sánchez et al. ${ }^{14}$ realizaram estudo descritivo comparativo, tendo por base questionários enviados a médicos generalistas que atuavam há mais de cinco anos na mesma unidade nos países escolhidos para o estudo. Como dito anteriormente, longitudinalidade para estes autores, cuja referência conceitual é Starfield, significa "acompanhamento dos distintos problemas de saúde de um paciente por um mesmo médico ao longo do tempo". A observação é que só registraram o ponto de vista do médico, desconsiderando a opinião do usuário.

Com relação aos resultados, os autores constataram que os países investigados apresentavam diferentes graus de longitudinalidade e de continuidade; Portugal obteve o melhor desempenho eFinlândia, o pior, por não dispor delista depacientes epelo menor nível de conhecimento por parte do profissional a respeito dos proble mas do paciente.

$M$ ainous et al. ${ }^{28}$, tendo Starfield como referência teórica, investigaram a relação entre confiança no profissional médico quando a fonte regular decuidados era um único médico equando essa fonte era um serviço de APS. Para tal, conduziram inquérito com amostra aleatória de pacientes no Reino Unido enos Estados Unidos. Como resultado, destacaram que o acompanhamento por um mesmo médico ao longo do tempo está associado com aumento da confiança neste médico, mas não necessariamente em uma relação de causa e efeito. Em todo caso, a continuidade seria um nutriente para a confiança. Logo, deveria ser encorajada, inclusive com incentivos financeiros.

Para investigar a relação entre a continuidade do cuidado e a satisfação do paciente, Fan et al. ${ }^{29}$ realizaram estudo transversal, utilizando como fontes de informação inquérito com pacientes atendidos em clínicas de atenção primáriae registrosadministrativos desses serviços. Asquestões versavam sobre a satisfação com 0 atendimento e com outros aspectos do serviço oferecido. Tendo por referência os postulados de Saultz ${ }^{12}$, estes autores definiram continuidade do cuidado como "relação entre médico e paciente 
caracterizada por confiança e senso de responsabilidade". Os resultados, após ajustes por características dos pacientes (sociodemográficas e de estado de saúde) e dos provedores, também apontaram relação positiva.

Apesar da falta de uniformidade conceitual, todos os estudos já mencionados apresentam resultados que demonstram a relevância da longitudinalidade e/ou da continuidade do cuidado, independente das dimensões e variáveisutilizadas nas análises. A fim de corroborar os resultados positivos deste atributo, valecitar o estudo deGill e Mainous ${ }^{30}$ e também duas revisões sistemáticas, uma de autoria de Saultz e Albredai wi ${ }^{31}$, que focaliza a relação da oferta de longitudinalidade com a satisfação do paciente, e a outra, de Cabana e $\mathrm{Jee}^{32}$, que destaca a relação do referido atributo com a qualidade do cuidado (Quadro 2).

Gill e M ainous ${ }^{30}$ investigaram a associação entre continuidade do cuidado eepisódios de internação a partir de um estudo longitudinal que considerou um período de dois anos. Este estudo envolveu cerca de 13.000 beneficiários do M edicaid. Embora esses autores não tenham utilizado o termo longitudinalidade, interpretaram "continuidade" como acompanhamento por um mesmo provedor ao longo do tempo, tendo Starfield como referência conceitual.

Gill e Mainous ${ }^{30}$ encontraram associação positiva entre continuidade com o mesmo provedor e menores taxas de internação para condições crônicas sensíveis ao tratamento ambulatorial. Mas para as condições agudas passíveis de tratamento ambulatorial, estes autores não encontraram associação positiva significativa.

A revisão sistemática realizada por Saultz e Albredaiwi ${ }^{31}$ contemplou trinta artigos originais que investigaram a relação entre satisfação do paciente e continuidade interpessoal. A base conceitual para o termo foi a revisão sistemática de Saultz ${ }^{12}$, realizada em 2003 ejá mencionada anteriormente (Quadro 1). 0 propósito da análise foi determinar a qualidade da evidência da satisfação do paciente, tendo por base os métodos utilizados nos estudos. Como conclusão, estes autores apontam que a pesquisa sobre continuidade permanecelimitada por diferentes definições etécnicas de medida, mas com evidências de que a continuidade interpessoal é importante para pacientes e médicos. $\mathrm{N}$ a opinião dos autores, tais evidências seriam suficientes para que a longitudinalidadena atenção primária fosselevada em consideração nas reformas de sistemas de saúde.

Com objetivo de investigar o efeito da continuidade na qualidade do cuidado, Cabana eJ $e^{32}$ conduziram revisão sistemática contemplando dezoito artigos, dos quais doze eram estudos transversais, cinco estudos de coorte eum ensaio randomizado. Ressalta-se que o estudo de Gill e $M$ ainous ${ }^{30}$ é um dos estudos de coorte contemplados nesta revisão.

O termo utilizado foi "continuidade sustentada do cuidado", tendo por referência os postulados de Starfield. Quanto aos critérios de seleção dos artigos, foram excluídos estudos que envolviam hospitalização anterior ou posterior, que não tinham um período padronizado para a medição e que a medida para o estudo era obtida por questões que não estivessem de acordo com o conceito de longitudinalidade assumido pelos autores da revisão. Cabana e Jee $e^{32}$ encontraram forte associação entre a longitudinalidade e benefícios relacionados à situação de saúde dos pacientes. Dentre os resultados, destacaram a diminuição da taxa de internação e do atendimento de emergência, melhor receptividade dos cuidados preventivos e melhor comunicação.

\section{Discussão}

Considerações teóricas

para o estudo da longitudinalidade

No presente estudo, a partir da bibliografia revisada, constata-se que três autores são citados como referência conceitual para 0 atributo em questão, a saber, Institute of M edicine da Academia Nacional de Ciências deWashington ${ }^{19}$ (autor denatureza institucional, definido a partir de painel de especialistas); I an R. M cW hinney ${ }^{17}$ eB. Starfield ${ }^{5}$. Estes autores apresentam duas percepções nucleares, quedizem respeito à semelhança entre os termos longitudinalidade e continuidade do cuidado.

Para os dois primeiros autores, Institute of $M$ edicine ${ }^{19} \mathrm{e} M$ cW hinney ${ }^{18}$, os termos longitudinalidadee continuidade do cuidado seriam similares; já para Starfield ${ }^{5}$, haveria diferenças conceituais. Um outro grupo de autores (Saultz ${ }^{12}$, H aggerty et al..$^{20}$ e Freeman e H jortdahl ${ }^{21}$ ) acrescenta elementos à definição dos termos utilizados pelos autores do primeiro conjunto.

Em termos operacionais, todavia, na literatura especializada internacional, o termo continuidade do cuidado tem sido utilizado com sentido semelhante, mas não idêntico, ao de longitudinalidade. Ou seja, a maioria dos autores, ao conduzir seus estudos utilizando o termo continuidade do cuidado, considera o significado do atributo 
da longitudinalidade empregado por Starfield ${ }^{5}$, principal menteno quediz respeito à identificação e utilização de uma fonte regular de cuidado de atenção primária, o que pressupõe a existência de vínculo longitudinal. No entanto, outros aspectos do atributo, como as características da relação entre profissional e paciente, não são consenso.

$\mathrm{N}$ a literatura especializada brasileira, a palavra "Iongitudinalidade" não é usual. Em trabaIhos avaliativos que fazem referência à Starfield, as palavras vínculo e continuidade têm sido utilizadas em vez de longitudinalidade. M as tais opções podem restringir o significado do atributo à existência de uma relação entre usuário e profissionais de atenção primária, sem a preocupação com as outras possíveis dimensões do atributo. Nesse sentido, a proposta aqui apresentada éque, naliteratura referente à APS no Brasil, façamosa opção pela utilização do termo vínculo longitudinal, definindo-o como "relação terapêutica estabelecida entre paciente e profissionais da equipe de APS, que se traduz no reconhecimento e utilização da unidade básica de saúde como fonte regular de cuidado ao longo do tempo". Para compor tal definição, seria necessário assumir algumas das dimensões identificadas pelos autores aqui revisados. Tais dimensões são apresentadas e discutidas a seguir.

Ressalta-se que a opção pela utilização do referido termo e sua respectiva definição está em acordo com o contexto da APS no Brasil, cuja tradição é de divisão de responsabilidades com uma equipe, embora o profissional médico seja imprescindível. 0 vínculo com profissionais da equipe em vez do vínculo com um único médico traz como uma das vantagens maior possibilidade de atender ao atributo da integralidade ${ }^{5}$.

\section{As dimensões do atributo}

da longitudinalidade/vínculo longitudinal

A partir dos estudos aqui revisados, podemos concluir que três elementos são imprescindíveis na composição do referido atributo: a existência e o reconhecimento de uma fonte regular de cuidados de atenção primária, o estabelecimento de vínculo terapêutico duradouro entre os pacientes e os profissionais de saúde da equipe local e, ainda, a continuidade informacional. Logo, tais elementos podem ser identificados como dimensões do atributo em questão. A discussão a seguir busca contextualizar, de forma sucinta, cada um desses elementos no âmbito da atenção primária na atual configuração do sistema público de saúde brasileiro.
Fonte regular

de cuidados de atenção primária

A identificação deuma fonteregular decuidados pressupõe que a população deve reconhecer a unidade básica como referência habitual para 0 atendimento da maioria das necessidades de saúde ${ }^{5}$. Tal identificação por parte da população depende em primeiro plano da oferta e disponibilidade dessa fonte, que deve estar em consonância, em termos qualitativos e quantitativos, com as necessidades de saúde da população local.

Nesse sentido, pode-se afirmar que os incentivos governamentais para a implantação de equipes do Programa de Saúde da Família (PSF), sem dúvida, promoveram ampliação da oferta de atenção primária na maioria dosmunicípios brasileiros. Torna-se pertinente, no entanto, investigar se existe por parte da população adscrita o real reconhecimento das unidades de PSF como fonte regular de cuidados ou se a utilização da unidadeésimplesmentepela restrição deopções, o que poderia implicar a não adesão às recomendações terapêuticas, e ainda a busca de serviços de emergência para tratamento de agravos passíveis de intervenção pela equipe deAPS.

Estabelecimento de vínculo duradouro (relação interpessoal)

O vínculo duradouro, segundo a literatura revisada, é sinônimo de uma relação terapêutica ao longo do tempo ${ }^{20}$, ou ainda, de relação interpessoal contínua entre paciente e cuidador, caracterizada por confiança e responsabilidade ${ }^{12}$. No contexto brasileiro, a Política Nacional de Atenção Básica ${ }^{33}$ explicita o vínculo entreas equipes de atenção primária e a população adscrita como um dos princípios deste nível de atenção.

Em todo caso, vínculo pressupõe em primeiro plano uma boa relação médico-paciente (no caso do PSF, profissionais desaúde paciente). Esta relação, por sua vez, remete ao campo da bioéti$\mathrm{ca}$, pois envolvequestões como poder edependência entresujeitos com diferentesníveis deinformação, além de práticas que podem levar a danos. N esse aspecto, vale lembrar que, para Caprara e Rodrigues ${ }^{34}$, uma boa relação médico-paciente envolve confiança, padrão de comunicação, consideração dos problemas relatados pelo paciente e dos aspectos biopsicossociais do mesmo.

Segundo esses autores, o modelo biomédico, por ser centrado na doença e privilegiar a tecnologia, diminuiu o interesse pela experiência do paciente e por sua subjetividade. Sendo assim, neste modelo, o paciente não é percebido em sua integralidade. Suas queixas psicossociais, quan- 
do ouvidas, não são levadas em consideração. Além do mais, não se promove a autonomia do paciente, uma vez que não há preocupação em esclarecer ao sujeito do cuidado o diagnóstico e as possibilidades terapêuticas. A princípio, a mudança de modelo assistencial estabelecidanos marcos legais que regulam o SUS deveria levar à superação de tais características no âmbito da atenção básica ${ }^{35}$.

Em se tratando das estratégias de avaliação do vínculo entre os usuários e a equipe de APS, dadas as características envolvidas, há de se considerar a opinião do paciente. Por outro lado, como afirmam Nutting et al. ${ }^{27}$, o vínculo com uma equipe de APS parece não ser interessante para determinados grupos, como os jovens, pessoas saudáveis e/ou pessoas de melhor poder aquisitivo. Ou seja, estes grupos podem não valorizar o vínculo como critério de qualidade na APS tanto quanto grupos populacionais considerados mais vulneráveis, como idosos, portadores de condições crônicas e grupos populacionais de baixo poder aquisitivo.

\section{Continuidade informacional}

A continuidade informacional, considerada a base da longitudinalidade $\mathrm{e}^{12,20}$, diz respeito à qualidade dos registros em saúde, seu manuseio e disponibilização, de forma a favorecer o acúmulo deconhecimento sobre o paciente por parte da equipe de saúde. Este acúmulo de conhecimento, que é dinâmico, deve orientar a conduta terapêutica e favorecer a avaliação da mesma.

$\mathrm{Na}$ atenção primária, os registros em saúde devem contemplar não só aspectos clínicos, mas também características sociodemográficas, valores e situação familiar do paciente ${ }^{20}$. Estas características interferem na condição de saúde do paciente e também podem constituir-se em empecilho na adesão deste às recomendações dos profissionais de saúde.

No que refere à continuidade informacional, o desenvolvimento e/ou implantação de novas tecnologias de informação por parte da gestão municipal e da coordenação da unidade pode ser um aliado. Tais instrumentos agilizam as etapas deregistro, processamento edisponibilização das informações em saúde. M as critérios como suficiência e confiabilidade são de responsabilidade dos profissionais que realizam os atendimentos, posto que a fonte do dado é quase sempre o pacienteem seu contato com a equipe de saúde. No Brasil, no âmbito da atenção primária, estudos que avaliem a continuidadeinformacional ainda são raros ou pouco difundidos.
Eleição de variáveis e de fonte de dados para o estudo da longitudinalidade

Os estudos sistematizados pela presente revisão que buscaram mensurar os resultados do atributo em questão utilizaram como fonte de informação dados administrativos, observação direta, inquéritos com pacientes einquéritos com profissionais, dentreoutros (Quadro 2). Asquestões de estudo, em sua maioria, diziam respeito à identificação da fonte regular decuidados em sua relação com a satisfação do paciente ou com supostos benefícios. N esse aspecto, a observação de Saultz ${ }^{12}$ é que alguns indicadores utilizados, que se referem à quantidade e continuidade do atendimento com o mesmo provedor, medem a continuidade longitudinal, mas não a continuidade interpessoal. Ou seja, não seriam sensíveis para avaliar as características da relação profissional de saúde paciente.

0 atendimento aos atributos descritos por Starfield ${ }^{5}$ tem servido como base para medir 0 alcance equalidade da APS. N esse aspecto, 0 instrumento elaborado por uma equipe da Universidade Johns H opkins (Primary Care Assessment Tools - PCAT) foi testado por vários autores 7,8 , inclusiveno Brasi ${ }^{9,10}$. N as duas experiências norte-americanas revisadas de testagem do instrumento, a longitudinalidadefoi considerada como atributo-chave, uma vez que a identificação da fonte regular de cuidados foi o ponto de partida da investigação, seguida de questões relativas à natureza do relacionamento do usuário com a referida fonte de cuidados ${ }^{7,8}$.

H arzheim et al. ${ }^{9}$ adaptaram e buscaram validar esse instrumento em um município do Sul do Brasil, tendo como fonte de dados entrevistas com responsáveis por crianças cadastradas em uma unidade básica de saúde. Nessa experiência, a opção foi pela utilização do termo "continuidade", embora a adaptação das questões do referido tópico tenha privilegiado a investigação da relação equipe-paciente.

Almeida e $M$ acinko ${ }^{10}$ também testaram esse instrumento em um município brasileiro localizado no Estado do Rio de Janeiro. Os entrevistados foram gestores e coordenadores da atenção primária, além de profissionais de saúde - considerados como informantes-chaves -, e ainda amostra de usuários.

As questões que compõe o PCAT foram validadas pelos autores que testaram o instrumento, o que legitima sua utilização para avaliar a APS, considerando os quatro atributos identificados por Starfield. M as, ao afirmar a longitudi- 
nalidade/vínculo longitudinal como característica central da APS, dada a sua relação com a efetividade neste nível assistencial, a sugestão é que utilizemos apenas este atributo para avaliar a qualidade da APS. Sendo assim, a proposta aqui apresentada é de construção de roteiro a partir deaproveitamento e adaptação deal gumas questões que compõe o PCAT, previamente testadas por Almeida e M acinko, acrescentando-senovas variáveis e questões, em consonância com as dimensões identificadas para 0 atributo.

0 referido roteiro (Q uadro 3) écomposto de quinze questões, sendo nove adaptadas da versão brasileira do PCAT e seis criadas a partir da presente revisão, quesão as denúmero $2,4,7,12$, 13 e 14 .

\section{Consideraçõesfinais}

O SU S avançou no processo de reorganização da atenção básica, inclusive com a ampliação da cobertura eimplementação denovos modelosassis- tenciais. M as, melhorar a qualidade dos serviços prestados nestenível de atenção ainda éum desafio. N esse aspecto, posto que estudos relacionam a longitudinalidade/vínculo longitudinal com re sultados positivos, o reconhecimento deste atributo como característica central da APS em nosso país éoportuno e deve ser almejado eavaliado.

Por outro lado, o fato do termo longitudinalidade ainda não ser usual na literatura brasileira e a existência de discordâncias com relação aos quesitos que compõem o referido atributo na literatura internacional permitem a adoção de dimensões em acordo com os princípios organizativos do sistema público de saúde do nosso país, desde que esteja em consonância com os pressupostos dos autores que discutem a temática.

A proposta aqui apresentada constitui-se em um roteiro de questões, as quais poderão ser adaptadas e direcionadas para realização de inquéritos com diferentes atores ( diretores de unidades, profissionais ou grupo de pacientes). N essa proposta, as opções de resposta não foram contempladas, mas a utilização de questionário

Quadro 3. Proposta de questões para investigação do vínculo longitudinal a partir das dimensões do atributo.

- Identificação ou reconhecimento da fonte regular de cuidados:

1. A unidade possui adscrição de clientela?

2. A unidade é reconhecida pela população adscrita como local de atenção para antigos e novos problemas de saúde?

- Relação interpessoal

3. 0 paciente é atendido regularmente pelo mesmo médico e/ou enfermeiro nas consultas de rotina?

4. Os profissionais que atendem o paciente conhecem o histórico familiar e social do paciente?

5. No caso de dúvida sobre tratamento, o paciente consegue falar com o profissional que 0 atende regularmente?

6. Durante 0 atendimento, há tempo suficiente para que os pacientes explicitem suas dúvidas, queixas e preocupações?

7. As dúvidas, preocupações e queixas do paciente são valorizadas? São registradas no prontuário?

8. 0 profissional se expressa com clareza, de forma que o paciente entenda?

9. Há espaço para que o paciente discuta seu tratamento e tome decisões junto com o profissional?

. Continuidade informacional

10. Os profissionais de saúde utilizam o prontuário nos atendimentos?

11. Os profissionais de saúde estão informados sobre todos os medicamentos utilizados pelo paciente?

12. Os profissionais de saúde estão informados sobre os exames realizados pelo paciente?

13. Em caso de encaminhamento para especialista, este profissional recebe informações registradas da unidade que atende o paciente regularmente?

14. No caso de referência para consulta com especialistas ou exames externos, há retorno dos resultados para o clínico que atende o paciente regularmente?

15. Os profissionais de saúde são informados quando um paciente não consegue obter o medicamento prescrito?

Fonte: adaptado da versão brasileira do PCA-tools testado por Almeida e M acinko. 
fechado facilitaria o tratamento dos dados e permitiria a utilização de estratégias de avaliação rápida com participação de grupos de pacientes.

É importante considerar que, embora o estabelecimento de vínculo longitudinal esteja no âmbito da prática do profissional, 0 atendimento a tal atributo só será possível se for uma prioridade da gestão, na medida em que envolve questões como a oferta adequada de serviços de APS e mecanismos defixação do profissional na unidade de saúde. Neste sentido, a implementação de práticas avaliativas e de monitoramento do referido atributo surtirá melhores efeitos se estiver no escopo de interesse da gestão.

\section{Colaboradores}

EM Cunha trabalhou na concepção teórica, elaboração e redação final do texto; L Giovanella participou da concepção teórica e revisão final do texto.
Referências

1. Macinko J, Starfied B, Shi L. The Contribution of Primary Care Systems to $\mathrm{H}$ ealth Outcomes within Organization for Economic Cooperation and Development (OCDE) Countries. HSR 2003; 38(3):831-865.

2. Starfield B, Shi L, Macinko J. Contribution of Primary Care to $\mathrm{H}$ ealth Systems and $\mathrm{H}$ ealth. The M ilbank Q 2005; 83 (3):457-502.

3. World Health Organization. What are the advantages and disadvantages of restructuring a health care system to be more focused on primary care services? Geneva: WHO; 2004.

4. Giovanella L. A atenção primária à saúde nos países da União Européia: configurações e reformas organizacionais na década de 1990. Cad Saude Publica 2006; 22(5):951-963.

5. Starfield B. Atenção primária: equilíbrio entre necessidades de saúde, serviços e tecnologia. Brasília: Unesco/M inistério da Saúde; 2002.

6. Shi L. Type of Health Insurance and the Quality of Primary Care Experience. Am J Public Health 2000; 90(12):1848-1855

7. Cassady CE, Starfield B, Hurt ado MP, Bark RA, Nunda JP, Freedenberg LA. M easuring consumer experiences with primary care. Pediatrics [periódico na Internet] 2000 [acessado 2006 jul 25];105:[ cerca de 6 p.]. Disponível em: http://www.jhsph.edu/bin/ w/u/M easuring_consumer_experiences.pdf

8. Leiyushi DRPH, Starfield B, Xu J. Validating the Adult Primary Care Assessment Tool. J. Fam. Pract. 2001; 50:161-175.

9. Harzhein E, Starfield B, Rajmil L, Stein AT. Consistência e interna e confiabilidade da versão em português do instrumento de avaliação primária (PCATool-Brasil) para serviços de saúde infantil. Cad Saude Publica 2006; 22(8):1647-1659. 
10. Almeida C, Macinko J. Validação de uma metodologia de avaliação rápida das características organizacionais e do desempenho dos serviços de atenção básica do sistema único de saúde (SUS) em nível local. Brasília: OPAS/M inistério da Saúde; 2006.

11. Gérvas J, Fernández MP. El fundamento científico de la función de filtro del médico general. Rev. bras. epidemiol. 2005; 8(2):205-218.

12. Saultz JW. Defining and M easuring Interpersonal Continuity of Care. Ann Fam M ed 2003; 1(3):134145.

13. Ortún V, Gérvas J. Fundamentos y eficiencia de la atención médica primaria. Med Clin (Barc) 1996; 106:97-102.

14. Pastor-Sánchez R, M iras AL, Fernández MP, Camacho RG. Continuidad $Y$ Longitudinalidad en M edicina General en cuatro países Europeos. Rev Esp Salud Pública 1997; 71:479-485.

15. Giovanella L, Escorel S, M endonça M HM. Porta de entrada pela atenção básica? Integração à rede de serviços de saúde. Saúde em Debate 2003; 27(65):278-289.

16. Mendes EV. Reflexões sobre a NOAS SUS 01/2002. [Mimeo].

17. Brasil. M inistério da Saúde. Secretaria de Atenção à Saúde. Departamento de Atenção Básica. Avaliação da Atenção Básica em Saúde: caminhos da institucionalização. Brasília: Ministério da Saúde; 2005.

18. M CW hinney IR. The principles of family medicine. In: M cWhinney IR, editor. A Textbook of Family $M$ edicine. $2^{\text {nd }}$ ed. New York: Oxford University Press; 1997.

19. Committee on the future of primary care. Primary care: America's health in a new era. $1^{\text {st }}$ ed. Washington, D.C.: Institute of Medicine/ $\mathrm{National} \mathrm{Academy} \mathrm{of}$ Sciences; 1996.

20. Haggerty JL, Reid RJ, Freeman GK, Starfield BH, Adair CE, M cKendry R. Continuity of care: a multidisciplinary review. BM J [periódico na Internet] 2003 [acessado 2007 jan 18]; 327:[cerca de 3 p.]. Disponível em: http://www.bmj.com/content/327/ 7425/1219.full

21. Freeman G, Hojordahl P. What future for continuity of care in general practice? BMJ [periódico na Internet] 1997 [acessado 2007 jan 18]; 314: [cerca de 1 p.]. Disponível em: http://www.bmj.com/content/314/ 7098/1870.full

22. Guthrie B, Whike S. Does continuity in General Practice really matter? BMJ [periódico na Internet] 2000 [acessado 2007 jan 18];321:[cerca de 3 p.]. Disponível em: http://www.bmj.com/content/321/ 7263/734.full

23. World Health Organization. Continuity of care in changing health care systems. Geneva: WHO; 1992.

24. Christakis DA. Continuity of care: Process or outcome. Ann Fam M ed 2003; 1(3):131-133.

25. Roberge D, Beaulieu MD, Haddad S, Lebeau R, Pineault R. Loyalty to the regular care provider: patients' and physicians' views. Family Practice [periódico na Internet] 2001 [acessado 2007 jan 17];18(1):[cerca de 6 p.]. Disponível em: http:// www.periodicos.capes.gov.br
26. De M aeseneer JM, De Prins L, Gosset C, Heyerick J. Provider continuity in family medicine: does it make a difference for total health care costs? Ann Fam M ed 2003; 1(3):144-148.

27. Nutting PA, Goodwin MA, Flocke SA, Zyzandki SJ, Stage KC. Continuity of primary care: to whom does et matter and when? Ann Fam Med [periódico na Internet] 2003 [acessado 2007 jan 17];1:[cerca de 8 p.]. Disponível em: http://www.periodicos.capes. gov.br

28. Mainous AG, Baker R, Love M M, Gray DP, Gill JM. Continuity of care and trust in one's physician: evidence from Primary Care in the United States and United Kingdom. Family M edicine [periódico na Internet] 2001 [acessado 2007 jan 17];33(1):[cerca de 6 p.]. Disponível em: http://www.periodicos.capes. gov.br

29. Fan VS, Burman M, MCDonell MB, Finn SD. Continuity of care and other determinants of patient satisfaction with primary care. JGIM [periódico na Internet] 2005 [acessado 2007 jan];20(3):[cerca de 8 p.]. Disponível em: http://www.periodicos.capes. gov.br

30. Gill JM, Mainous AG. The role of provider continuity in preventing hospitalizations. Arch Fam M ed [periódico na Internet] 1998 [acessado 2007 jan 17];7:[cerca de 6 p.]. Disponível em: http:// archfami.ama-assn.org/cgi/reprint/7/4/352.pdf

31. Saultz JW, Albedaiwi W. Interpersonal continuity of care and patient satisfaction. Annals of M edicine [periódico na Internet] 2004 [acessado 2007 jan];2(5):[cerca de 7 p.]. Disponível em: http:// www.periodicos.capes.gov.br

32. Cabana MD, Jee SH. Does continuity of care improves patient outcomes? J Fam Pract. [periódico na Internet] 2004 [acessado 2007 jan 17]; 53(12):[cerca de 7 p.]. Disponível em: http://www.periodicos. capes.gov.br

33. Brasil. M inistério da Saúde. Secretaria de Atenção à saúde. Departamento de Atenção Básica. Política Nacional de Atenção Básica. Brasília: Ministério da Saúde; 2006.

34. Caprara A, Rodrigues J. A relação assimétrica médico-paciente: repensando o vínculo terapêutico. Cien Saude Cole 2004; 9(1):139-146.

35. M endes EV. A Atenção Primária no SUS. Fortaleza: Escola de Saúde Pública do Ceará; 2002.

Artigo apresentado em 29/09/2008

A provado em 09/02/2009

Versão final apresentada em 09/03/2009 